\title{
ESTADO DE CONSERVACIÓN DE LOS SUELOS DE LA CUENCA DEL EMBALSE JOAQUÍN COSTA
}

\author{
José A. Martínez-Casasnovas, Rosa Ma Poch \\ Departament de Medi Ambient i Ciències del Sòl (E.T.S.E.A.).Universitat de Lleida. Av. A. Rovira Roure, 177. \\ 25 198-Lleida.
}

\section{RESUMEN}

Con el objetivo de reducir el grado de colmatación del embalse Joaquín Costa se realizó un estudio para evaluar el estado de conservación de la cuenca de $1500 \mathrm{~km}^{2}$ que vierte al embalse, y proponer medidas de control de la erosión.

Se utilizó un enfoque multiescalar de acuerdo a las siguientes fases: (1) identificación de áreas críticas fuente de sedimentos utilizando información litológica y topográfica, (2) evaluación de la cubieria vegetal en esas áreas mediante imágenes de satélite, (3) cartografía de la erosión y aplicación de modelos de erosión en subcuencas medianas utilizando fotografías aéreas secuenciales y prospecciones semidetalladas de suelos en el campo y (4) propuestas de medidas de conservación en laderas y cursos de agua.

La zona más crítica fue una región afectada por badlands en el centro de la cuenca. Su contribución estimada a la colmatación del embalse es del orden de $0.5 \mathrm{hm}^{3} / \mathrm{año}$, lo cual representa una gran parte del grado de colmatación ( 18 hm ${ }^{3}$ en 20 años). Las medidas de control de la erosión que se proponen son diques semipermeables en los torrentes mas críticos y prácticas agrícolas de manejo en las áreas más contributivas.

Palabras clave: cuenca, cartografía de la erosión, SIG, badlands, medidas de conservación de suelos

\begin{abstract}
Within the objective of preventing the siltation of the Joaquin Costa reservoir, a study was carried out in order to assess the conservation status of the watershed, which has un area of $1500 \mathrm{~km}^{2}$, and to propose measures on control erosion accordingly.

An increasing scale approach was used according to the following phases: (I) identification of the critical areas of sediment source using lithological and topographical information, (2) evaluation of the vegetation cover in these areas by satellite imagery, (3) erosion mapping and application of erosion models at medium sub-watershed scale using multi-temporal aerial photographs and semi-detailed field surveys and (4) proposal of soil conservation measures on slopes and water courses accordingly.

The most critical area was a badland-affected region in the center of the watershed. Its estimated contribution to the siltation was about $0.5 \mathrm{Hm}^{3} /$ year, which accounts for most of the $18 \mathrm{hm}^{3}$ of sediments deposited during 20 years in the reservoir: The proposed erosion control measures consist of grade control structures as gabion dams; and agricultural management practices in the most critical areas.
\end{abstract}

Key words: watershed, soil erosion mapping, GIS, badlands, soil conservation measure. 1 .

\section{INTRODUCCIÓN}

Dentro de los componentes del estudio de los impactos del vaciado del embalse Joaquín Costa, el diagnóstico del estado de conservación de la cuenca es el Único que no se ve afectado por el vaciado, ya que su objetivo es la localización de las áreas fuente de sedimentos y la propuesta de medidas correctoras para disminuir dichos aportes. Una estrategia realista para conseguirlo no puede pretender actuar en la totalidad de la cuenca, debido a su gran extensión. El enfoque a utilizar tiene que optimizar el coste de las medidas que se propongan frente a los beneficios derivados de su aplicación: a igual coste, se reducirá más la aportación de sedimentos actuando puntualmente en las zonas más críticas que planteando medidas en áreas extensas.

La cartografía de la erosión a pequeña escala como instrumento de gestión de grandes cuencas se enfrenta al problema de encontrar un compromiso entre la inversión del estudio y la validez de la cartografía. Clásicamente los estudios detallados de la erosión requieren largos años de medidas en el campo. Con todo, la representatividad de los resultados no está asegurada debido a los múltiples factores que intervienen a lo largo del proceso de la erosión y sedimentación. En el otro extremo, la aplicación de modelos empíricos de cuantificación de la erosión, como la USLE (Por- 
ta et al., 1994), presentan problemas debido a su falta de adecuación cuando se aplican en cuencas a pequeña escala, ya que no reflejan todo el abanico de posibles procesos erosivos, como deslizamientos, cárcavas o barrancos, por otra parte muy importantes en la cuenca de estudio.

\section{OBJETIVOS}

El estudio ha seguido un enfoque multinivel (a diferentes escalas) con los siguientes objetivos:

- Primera fase: Proponer y validar un modelo de identificación cualitativa de zonas críticas respecto a la emisión de sedimentos y localizar las zonas de mayor contribución potencial al aterramiento del embalse Joaquín Costa.

- Segunda fase:

- Evaluar el grado de erosión real en las zonas críticas obtenidas en la primera fase,

- Obtener información sobre la dinámica de la erosión en badlands, barrancos y cárcavas.

- Tercera fase: Proponer medidas concretas de actuación en áreas modelo.

\section{METODOLOGÍA GENERAL}

Para conseguir los objetivos de cada fase se han utilizado distintos tipos de información, métodos y escalas, resumidos en la Tabla 1. La información generada en cada una de las fases es utilizada como datos de partida para la siguiente.

\section{Identificación de zonas emisoras de sedimen- tos}

La información utilizada en esta primera fase ha sido la densidad de drenaje y la litología, información fácil de obtener y consistente geográficamente. La densidad de drenaje definida por Horton (1945), que permite medir el grado de disección de una cuenca. También indica la escorrentía superficial que se produce en la cuenca, por el hecho que a más pendiente aumenta la velocidad de la escorrentía y por lo tanto la capacidad transportadora de sedimentos (Morgan 1973; Gregory \& Gardner 1975; Dunne \& Leopold 1978). La litología, por otra parte, determina la resistencia de los materiales a erosionarse, así como su capacidad de infiltración que condiciona la generación de escorrentía (Del Val 1989).Existen diversos índices para medir la erosionabilidad del suelo. Uno de ellos relaciona directamente esta variable con el substrato litológico (FAO 1977, cit. MOPT 1992).

Para la delimitación de la red y de las cuencas de drenaje se ha utilizado el mapa topográfico 1:100 000, mientras que la información litológica se ha extraído de los mapas geológicos 1:200 000 "Síntesis de la cartografía existente" n. 23, Huesca, 2" edición; y de los 1:50 000 "Serie Magna", hojas de Bielsa y Liena, del ITME.

Tabla 1. Metodología e información utilizada para las distintas fases del estudio. Methodological process and information used to carry out the differents parts of the study.

\begin{tabular}{|c|c|c|c|c|}
\hline Objetivos & Información de partida & Métodos & $\begin{array}{l}\text { Escala de } \\
\text { trabajo }\end{array}$ & $\begin{array}{l}\text { Escala de } \\
\text { presentación }\end{array}$ \\
\hline $\begin{array}{l}\text { Identificación de áreas } \\
\text { fuente de sedimentos }\end{array}$ & $\begin{array}{l}\text {-topografía } \\
\text {-geología } \\
\text {-hidrografía }\end{array}$ & $\begin{array}{l}\text {-sistemas de información } \\
\text { geográfica (SIG) } \\
\text {-reconocimiento general de campo }\end{array}$ & $1: 100.000$ & $1: 300.000$ \\
\hline $\begin{array}{l}\text { Evaluación del grado de } \\
\text { erosión y dinámica de las } \\
\text { principales áreas fuente }\end{array}$ & $\begin{array}{l}\text {-climatología } \\
\text {-imágenes de satélite } \\
\text {-fotografías secuenciadas }\end{array}$ & $\begin{array}{l}\text {-fotointerpretación multitemporal } \\
\text {-clasificación imágenes de satélite } \\
\text {-reconocimiento semidetallado de } \\
\text { campo }\end{array}$ & $\begin{array}{l}1: 100.000 \\
1: 50.000 \\
1: 30.000\end{array}$ & $\begin{array}{l}1: 100.000 \\
1: 50.000\end{array}$ \\
\hline $\begin{array}{l}\text { Propuesta de medidas } \\
\text { correctoras (áreas modelo) }\end{array}$ & $\begin{array}{l}\text {-cartografía detallada } \\
\text {-fotografías aéreas }\end{array}$ & $\begin{array}{l}\text {-reconocimiento detallado de } \\
\text { campo }\end{array}$ & $\begin{array}{l}1: 30.000 \\
\mathrm{I}: 25.000\end{array}$ & $\begin{array}{l}1: 50.000 \\
I: 25.000\end{array}$ \\
\hline
\end{tabular}


Tabla 2. Porcentajes de área correspondientes a las distintas clases de riesgo de emisión de sedimentos para el embalse de Joaquín Costa. Areal percentages correspondent to the different sediment emission risk classes for the catchment of the Joaquín Costa reservoir.

\begin{tabular}{lcc}
\hline Clase de riesgo de emisión de Sedimentos & Área (ha) & Porcentaje (\%) \\
\hline l bajo & 43825.78 & 29.16 \\
2 moderado & 69353.41 & 46.15 \\
3 alto & 18944.71 & 12.60 \\
4 severo & 13293.44 & 8.85 \\
5 muy severo & 4867.88 & 3.24 \\
\hline
\end{tabular}

A cada una de las unidades del territorio resultantes se le asigna una clase de riesgo de emisión de sedimentos, en función del valor de la densidad de drenaje y de la litología. La valoración de estos dos parámetros se realiza mediante los criterios de Bucko \& Mazurova (1958, cit. Stroosnijder \& Eppink 1993 y Martínez-Casasnovas 1994) y de FAO (1977) respectivamente. El valor del coeficiente para delimitar la clase de riesgo de emisión de sedimentos se obtiene multiplicando ambos factores.

Los resultados numéricos de la aplicación del modelo se resumen en la Tabla 2. Según estos resultados, una cuarta parte de la cuenca presenta un alto riesgo de emisión de sedimentos o mayor (clases 3, 4 y 5).

Se pueden observar tres zonas repartidas por la cuenca que destacan por su alto riesgo. Estas zonas se han denominado zona norte, central y sur:

- La zona norte corresponde al valle entre el pico de Cotiella (1912 m) y la Sierra de Chía, afectando principalmente el barranco de Comallitera, que desagua al barranco de Barbarúens, en una zona parcialmente vegetada por bosques de coníferas. Predomina la clase de riesgo 4, asociada a un material litológico poco resistente, como son las margas y turbiditas.

- La parte central de la cuenca, situada al sur del Turbón (2492 m), ocupa los barrancos del Valle del Lierp, por el corredor de Merli, Bacamorta y La Foradada en el río Ésera, y los torrentes de Villacarlí y del Congustro en el río Isábena. Es una franja con valles excavados en margas con areniscas en las divisorias de aguas. La densidad de drenaje no es muy elevada.

- AI sur, alrededor de Laguarres y Lascuarre y sobre una mancha de conglomerados, se sitúan una serie de barrancos en una zona de uso agrí- cola. El elevado riesgo de emisión se debe a una densidad de drenaje elevada.

Las fotografías aéreas para la validación del modelo corresponden a distintos vuelos del Instituto Geográfico Nacional a escala aproximada 1:30 000. En la fotointerpretación de la cuenca se observaron tres formas de erosión significativas en cuanto a su importancia: cárcavas y barrancos (gullies), badlands y claros, cuya distribución se comparó con el mapa final. La fiabilidad global así obtenida resultó ser del $78.53 \%$.

\section{Evaluación del grado de erosión y dinámica en las zonas emisoras de sedimentos}

\section{Evaluación del grado de erosión.}

La segunda fase del estudio se localizó en el centro y sur de la cuenca, con el objetivo de obtener la distribución del grado de erosión real en las subcuencas.

La información utilizada fue la erosividad de la lluvia, la pendiente y la cubierta vegetal. La erosividad de la lluvia (Factor R de la USLE) se calculó a partir de series recientes de 9 estaciones. Las pendientes se clasificaron entre los valores de 2, 7, 15,30 y $45 \%$ obtenidas a partir de un Modelo Digital del Terreno (retícula $200 \mathrm{~m}$ ), mediante el programa Idrisi. La cubierta vegetal se evaluó a partir de una imagen Landsat TM de Julio-93, a una escala de 1:100 000. Se realizó una clasificación de la imagen mediante prospección de campo, fotointerpretación y comparación con el mapa de usos del suelo. La precisión del modelo fue de difícil comprobación debido a la heterogeneidad de la cubierta vegetal. Con todo, la precisión en las áreas de entrenamiento fue del $93.21 \%$ y fuera de ellas del $70 \%$.

Los factores de riesgo de la erosión se evaluaron cualitativamente y el riesgo de erosión real se 
Tabla 3. Áreas afectadas por las distintas clases de riesgo de erosión. Areas included in the different erosion risk class.

\begin{tabular}{llc}
\hline Clase de riesgo & Descripción & Superficie (ha) \\
\hline Nulo & Afloramientos de roca & 2739.6 \\
Bajo & Áreas con muy buena cubierta vegetal $(\%)$ & 60274.8 \\
Moderado & Áreas con buena cubierta vegetal y muy susceptibles, & \\
& o cubierta moderada y moderadamente susceptibles & 16748.7 \\
Alto & Áreas con baja cubierta vegetal, moderadamente susceptibles & 10815.6 \\
Severo & Áreas con baja cubierta y muy susceptibles & 9596.8 \\
Muy severo & Áreas con baja cubierta y altamente susceptibles & 1354.5 \\
\hline
\end{tabular}

definió de acuerdo al criterio de la Tabla 3, superponiendo los mapas correspondientes a cada factor. En esta tabla se encuentran también las superficies que ocupa cada clase de erosión.

El mapa resultante (Fig. 6 del anexo) muestra una gradación de la erosión así estimada de sur a norte en la cuenca. La parte sur tiene bajo riesgo debido a la baja amplitud del relieve y lluvia menos erosiva a pesar de su baja cubierta vegetal. En la parte norte el mayor recubrimiento también hace disminuir el riesgo. La zona de más riesgo es la de transición, que coincide con el afloramiento de bandas de calcilutitas donde se desarrollan los badlands.

\section{Caracterizacióny dinámica de badlands mediante el estudio multitemporal de fotografías aéreas y SIG}

\section{Caracterización de badlands}

El área de estudio de caracterización y dinámica de badlands se localiza en la zona central de la cuenca, donde se identificaron las áreas de mayor riesgo de emisión de sedimentos, tanto por la litología de los materiales que afloran (margas) como por su conexión a la red de drenaje principal de la cuenca (Fargas 1995; Fargas et al., 1996).

El área considerada tiene una extensión de 237 $\mathrm{km}^{2}$ y comprende las cuencas del río Rialbo y los barrancos de Espluga, Bacamorta, Carrasquero y arroyo de Villacarlí, todos ellos en las cercanías de Campo y el Turbón. En esta área se llevó a cabo una cartografía de las áreas ocupadas por badlands a partir de la interpretación de fotografías aéreas E. 1:30000 de 1984e interpretación visual de una imagen Landsat RGB 543, produciendo un mapa de cubierta vegetal y usos del suelos a E. $1: 50000$.
La caracterización de badlands se completó mediante la medida in situ de la erosión o deposición de materiales en las paredes de 6 tipos de badlands representativos. La erosión/deposición fue medida mensualmente (durante un año) respecto a un nivel de referencia inicial en estacas clavadas en la superficie.

La figura 7 del anexo muestra el mapa de tipos de badlands y cubierta vegetal del área estudiada. La mayor parte de las áreas está ocupada por vegetación arbórea o arbustiva bien desarrollada (42.5\%), mientras que $13.8 \%$ (3270 ha) de la superficie corresponde a áreas de badlands.

Se han distinguido 8 tipos de badlands según su amplitud de relieve, grado de ondulación de las paredes (como medida de su superficie específica) y grado de recubrimiento de la vegetación herbáceo-arbustiva desarrollada en sus paredes. De estos, el que mayor superficie representa es el tipo 1 (Fig. 7 del anexo), que se encuentran fundamentalmente en la parte orientada al sur y, por sus características, son los más activos y donde mayores tasas de producción de sedimentos se han registrado.

La tasa media de materiales que se movilizaron en las paredes de los badlands, para las condiciones del año 1996, fue de 302 Mg ha-' año-', con tasas máximas medidas mediante el método de las estacas de hasta $455 \mathrm{Mg}$ ha-' año-'. Estas cantidades son muy elevadas ya que el proceso de remoción de materiales se ve favorecido por los contrastes térmicos que se producen diariamente en esta zona prepirenaica. Esto produce la disgregación de los materiales en los primeros $\mathrm{cm}$ del afloramiento, facilitando su movilización por las lluvias.

El total de los materiales movilizados en los badlands del área de estudio se ha estimado en 
$987540 \mathrm{Mg}$ año-' en las 3270 ha cartografiadas $\left(0.604 \mathrm{hm}^{3}\right.$ año-'). Una gran parte de estos materiales, aproximadamente $0.5 \mathrm{hm}^{3}$ año-' según estimaciones aplicando coeficientes de transporte de sedimentos, son incorporados rápidamente a la red de drenaje a través de afluentes directos del Ésera e Isábena y finalmente acaban sedimentándose en su mayoría aguas abajo en el embalse Joaquín Costa. Esta cifra, aunque calculada a partir de datos relativos al año 1996, es indicativa de la alta contribución de las áreas de badlands al aterramiento en el embalse, sobre todo si tenemos en cuenta que en el momento del vaciado (1995) el volumen de aterramiento era aproximadamente de $18 \mathrm{hm}^{3}$.

\section{Dinámica de los badlands}

Dentro del área de estudio se eligió una subcuenca de $1.6 \mathrm{~km}^{2}$ donde se llevó a cabo el análisis de la dinámica de badlands. Se utilizaron fotografías aéreas de los años 1956 y 1984 (E. 1:50 000). En ambas se realizó la fotointerpretación de los badlands (límites y tipo) y otros usos del suelo y cubierta vegetal. El resultado de la fotointerpretación se restituyó mediante un proceso fototogramétrico, obteniendo dos coberturas vectoriales en formato digital con georeferenciación UTM y E. 1:10 000, conteniendo las delineaciones de la fotointerpretación. Posteriormente se les dio topología vectorial con el SIG ArcInfo y se analizaron mediante operaciones de superposición espacial y tabulación cruzada con los SIG ArcInfo 3.5 e Idrisi 2.0.

Los cambios producidos en el periodo de 28 años considerado se resumen en la Tabla 4 y se muestran en la Fig. 8 del anexo.
Los resultados obtenidos muestran que la tasa de crecimiento de las áreas de badlands es de aproximadamente entre $4.6 \mathrm{ha} / \mathrm{anno}$. El avance de estas áreas se produce por expansión de sus cabeceras a favor de los afloramientos de margas que existen en estas cuencas. Esta superficie es similar a la que supone el cambio entre áreas de badlands a otros tipos de usos o de cubierta vegetal (5.8 ha/año). Esto no supone una estabilización de los badlands por crecimiento de vegetación en sus paredes, sino que se debe a movimientos de tierra hechos por agricultores para recuperar terrenos de cultivo o por avance del badland hacia la cabecera. Esto indica la dimensión geológica de la erosión por crecimiento de badlands, que continuará hasta el agotamiento del afloramiento de margas.

También es significativo, respecto a la erosión, el mayor porcentaje de cambio que existe en la zona entre tipos de cubierta vegetal que suponen una menor protección del suelo $(8.4 \%$ de la superficie respecto a $3.8 \%$ de la superficie en la que hay un cambio hacia mayor protección). Esto indica que, no solamente se han de tomar medidas de control para el avance de badlands o del control de los sedimentos generados en ellos, sino que también habrá que implementar medidas de conservación en parcelas agrícolas, control del pastoreo y repoblación forestal en estas cuencas.

\section{Estrategias en conservación de suelos: Aplicación a áreas modelo}

El objetivo de esta fase fue obtener mapas detallados de zonas con problemas de erosión para ubicar y diseñar medidas de conservación de suelos. Ante la imposibilidad de actuar en toda la

Tabla 4. Superficies y porcentajes de los cambios producidos en una parte de la cuenca del río Rialbo (Campo. Huesca) de $1.6 \mathrm{~km} 2$ entre 1956 y 1984. Areas and percentajes of changes produced in a part of the Rio Rialbo catchment (Campo, Huesca) of $1.6 \mathrm{~km} 2$ between 1956 and 1984.

\begin{tabular}{|c|c|c|}
\hline Tipo de cambios & Superficie (ha) & Porcentaje (\%) \\
\hline Sin cambios & 1003 & 63.9 \\
\hline Cambio de barranco/badland a otro tipo de cubierta vegetal o uso del suelo & 156.7 & 9.9 \\
\hline Cambio de otro tipo de cubierta vegetal o uso del suelo $u$ barranco/badland & 125.7 & 8.0 \\
\hline Cambio entre tipos de barrancos/badlands & 91.1 & 5.8 \\
\hline Cambio entre usos del suelo o cubierta vegetal de mayor a menor protección del suelo & 131.4 & 8.4 \\
\hline Cambio entre usos del suelo o cubierta vegetal de menor a mayor protección del suelo & 60.2 & 3.8 \\
\hline
\end{tabular}


Tabla 5. Datos pluviométricos de la cuenca del Congustro $(\mathrm{Pen} \mathrm{mm})$. Rainfall data $(P$ in mm) of the Congustro catchment.

\begin{tabular}{llllllllllllll}
\hline & $\mathrm{E}$ & $\mathrm{F}$ & $\mathbf{M}$ & $\mathbf{A}$ & $\mathbf{M}$ & $\mathbf{J}$ & $\mathbf{J}$ & $\mathbf{A}$ & $\mathbf{S}$ & $\mathrm{O}$ & $\mathrm{N}$ & $\mathrm{D}$ & total \\
\hline P media & 48.7 & 47.1 & 48.5 & 70.9 & 89.5 & 78.4 & 46.2 & 61.3 & 74.8 & 71.9 & 72.7 & 56.9 & 767.5 \\
P 24 horas & 19.9 & 17.0 & 18.5 & 23.3 & 29.9 & 26.2 & 20.5 & 27.7 & 30.7 & 27.7 & 27.4 & 21.3 & \\
Días de 1luvia & 5 & 5 & 6 & 8 & 10 & 8 & 5 & 6 & 6 & 7 & 7 & 5 & 78 \\
\hline
\end{tabular}

cuenca se han escogido áreas modelo especialmente problemáticas según los resultados de las fases anteriores. Se muestran los resultados del estudio de la Cuenca del Congustro, ubicada en la parte central de la cuenca. Corresponde a un afluente del río Isábena y está excavada en calcilutitas altamente erosionables y que han evolucionado geomorfológicamente formando una activa red de cárcavas y badlands. Tiene una superficie de $20.26 \mathrm{~km}^{2}$ y un desnivel máximo de $870 \mathrm{~m}$ entre las cotas extremas. La pendiente media de la cuenca, representativa de la zona, es elevada (30.9\%), así como las pendientes medias de los cursos, que superan el 25\% llegando hasta cerca del $70 \%$, lo cual indica el alto grado de torrencialidad de los mismos. El análisis de la ramificación de la red de drenaje arroja un valor medio de confluencia de 4.11 , propio de una fuerte potencialidad erosiva, escorrentía rápida y tendencia de los tributarios a dirigirse a un solo curso principal.

El régimen de precipitaciones muestra que la época más erosiva es en otoño (Tabla 5 ) donde altas pluviometrías se concentran en pocos días de lluvia.

El estudio de las precipitaciones extremas se ha realizado a partir de una serie de 36 años para la cuenca. El ajuste a la función de Gumbel muestra que una precipitación diaria de $67.8 \mathrm{~mm}$ tiene una probabilidad anual del $10 \%$ (período de retorno de 10 años), mientras que una lluvia diaria de $98.2 \mathrm{~mm}$ se supera anualmente sólo con una probabilidad de $0 \mathrm{~S} \%$ (período de retorno de 200 años).

Los suelos de la cuenca están desarrollados sobre calcilutitas, calcáreas, areniscas y conglomerados eocenos, dispuestos en estratos buzados al S-SW. Geomorfológicamente se distinguen diversos niveles de glacis de erosión, en los cuales la red hidrográfica se ha ido encajando formando barrancos que delimitan superficies colgadas de pendientes entre 10 y $20 \%$ situadas a la altura media de la cuenca, actualmente ocupadas por campos de cultivo y pastos. La red hidrográfica en sí está encajada en las calcilutitas, coincidiendo con las zonas de más pendiente. No se observan procesos de incisión activos importantes en los cursos principales, por lo que los procesos geomorfológicos más activos son los movimientos de masa y erosión hídrica en laderas y en los badlands.

Los suelos de la Cuenca del Congustro se caracterizan por su escaso desarrollo, traducido en la ausencia de horizontes de diagnóstico. Se clasifican en su mayoría como Xerorthents (Soil Survey Stat, 1996). Corresponden a Xerorthents líticos en las laderas con calcáreas a poca profundidad (perfiles A-R), y Xerorthents típicos cuando están desarrollados sobre calcilutitas o coluvios de ladera en posiciones más estables (perfiles A-C o A-BwC). En general se trata de suelos básicos, pedregosos a muy pedregosos, de textura franco-limosa y excepcionalmente franco-arenosa en los desarrollados sobre coluvios de areniscas de la vertiente sur. En general son poco profundos y con contenidos en materia orgánica bajos $(<2 \%)$. Ambas características aumentan en las zonas de umbría, que son las que gozan de un recubrimiento vegetal mayor. Son suelos bien drenados, con capacidades de retención de agua limitadas y con estabilidad estructural moderada a baja.

Los suelos más erosionados corresponden, además de los badlands, a los de uso agrícola situados en los glacis, donde la erosión por regueros es muy activa; y a los suelos bajo matorral de la solana de la cuenca, con bajo recubrimiento vegetal.

La aplicación del modelo USLE (Wischmeier \& Smith 1978) para evaluar el material removido por erosión laminar y por regueros ha permitido conocer su órden de magnitud y distribución en la cuenca, así como los factores de riesgo que más afectan a la erosión de este tipo. Así, se aprecia que la erosividad de la lluvia, que oscila entre 


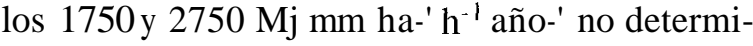
na zonas más o menos erosionables en la cuenca. Los suelos más erosionables $\left(\mathrm{K}=0.3 \mathrm{Mg} \mathrm{h} \mathrm{Mj}^{-1}\right.$ $\mathrm{mm}^{-1}$ ) son los de textura arenosa fina, pero se encuentran en zonas de umbría y gozan de mayor cubierta vegetal. Valores parecidos de $\mathrm{K}$ tienen los suelos de la zona sur de la cuenca, ocupados por cultivos en altas pendientes, por lo que el riesgo es mayor al carecer de protección durante una parte del año. La elevada erosionabilidad de los badlands $\left(\mathrm{K}=0.024 \mathrm{Mg} \mathrm{h} \mathrm{Mj}^{-1} \mathrm{~mm}^{-1}\right)$ se debe principalmente a su baja permeabilidad y bajo contenido en materia orgánica. La topografía afecta la erosión USLE de forma desigual: el factor LS, calculado para 17 subcuencas elementales oscila entre 2.2 y 8.7 en función de la longitud y pendiente de la ladera. El factor cubierta vegetal es el más variable: la mayor protección la ejercen los bosques densos $(\mathrm{C}=0.005)$ mientras que en las zonas más desfavorables, con poca vegetación o ocupadas por almendros, este factor aumenta unas 80 veces $(C=0.40)$. Las prácticas de conservación en la cuenca son escasas, y se limitan al laboreo según las curvas de nivel en algunas zonas ocupadas por cereal, las cuales reducen la erosión como máximo a la mitad.

Los valores de erosión en $\mathrm{Mg} \mathrm{ha}^{-1}$ año-' estimados según la USLE permiten clasificar los suelos de la cuenca en 5 clases de erosión (Fig. 9 del anexo). Los resultados indican que aproximadamente un $70 \%$ de la cuenca sufre pérdidas de suelo superiores a $20 \mathrm{Mg}$ ha-' año-' por erosión laminar y regueros, y en ella más de un tercio (37.5\%) de la superficie las pérdidas son mayores que $50 \mathrm{Mg} \mathrm{ha}^{-1}$ año-'. La alta conexión de las áreas erosionadas, las pocas zonas de sedimentación observadas en el campo y la presencia de cárcavas y barrancos cuya erosión no es evaluada por el modelo USLE ponen de manifiesto la gravedad de los procesos erosivos y la contribución a la colmatación del embalse de esta cuenca modelo.

Este comportamiento se refleja también tras la aplicación del modelo MUSLE (Onstad \& Foster 1975), que estima la exportación de sedimentos de la cuenca debidas a aguaceros tipo con distintos periodos de retorno en el punto de salida de la cuenca (Tabla 6). Aunque estos valores hay que interpretarlos con precaución, debido al empirismo del modelo, muestran que estas áreas, situadas en la parte media de la cuenca del embalse, explican por sí solas el grado de colmatación del embalse $\left(18 \mathrm{hm}^{3}\right)$ y por lo tantojustifican la aplicación de medidas de conservación en ellas.

De acuerdo con los procesos observados se han propuesto dos tipos de medidas de conservación de la cuenca: por una parte medidas hidrotécnicas de estabilización de márgenes de las cárcavas y badlands, consistentes en diques de consolidación situados en puntos críticos (a menudo en confluencia de los badlands con los cursos principales), donde la cuña de aterramiento que se forme estabilice su nivel de base. Los badlands más críticos en este sentido se han escogido en función del área de su cuenca afectada por la erosión, el grado de actividad de los procesos y su proximidad a los cursos principales. En el caso de la Cuenca del Congustro se han seleccionado dos puntos: el Barranco de Comacentre y el Barranco del Salt d'Aigua.

Por otra parte, y a la vista de los procesos en laderas se han determinado medidas fitotécnicas y agrotécnicas en función de la clasificación de la superficie de la Tabla 7, que se ha utilizado para

Tabla 6. Estimación de la exportación de sedimentos de la Cuenca del Congustro $\left(20.26 \mathrm{~km}^{2}\right)$ según el modelo MUSLE (Onsiad \& Foster 1975), considerando una densidad aparente de $1.5 \mathrm{Mg} / \mathrm{m} 3$. Estimation of exported sediments from the Congustro catchment (20.26 km ${ }^{2}$ ) calculated by application of the MUSLE (Onstad \& Foster 1975), considering a bulk density of $1.5 \mathrm{Mg} / \mathrm{m}^{3}$.

\begin{tabular}{cccc}
\hline $\begin{array}{c}\text { Período de retorno } \\
(\text { años })\end{array}$ & $\begin{array}{c}\text { Sedimentos } \\
\text { exportados }(\mathrm{Mg})\end{array}$ & $\begin{array}{c}\text { Volumen de } \\
\text { sedimentos (hm3. 10-3) }\end{array}$ & $\begin{array}{c}\text { Degradación específica } \\
\text { en todo el periodo (Mg/ha) }\end{array}$ \\
\hline 2 & 1250 & 0.83 & 6.17 \\
5 & 3225 & 2.1 & 15.92 \\
25 & 11543 & 1.1 & 56.97 \\
50 & 16451 & 1.0 & 81.20 \\
100 & 21840 & 14.6 & 107.80 \\
\hline
\end{tabular}


Tabla 7. Valores de erosión en los suelos de la Cuenca del Congustro y superficies afectadas. Soil erosion values in the Congustro catchment and affected surfaces.

\begin{tabular}{|c|c|c|}
\hline $\begin{array}{l}\text { Rango de erosión } \\
\text { USLE (Mg/ha.año) }\end{array}$ & Tipo de erosión & $\begin{array}{l}\text { Superficie afectada } \\
\text { en la cuenca }(\%)\end{array}$ \\
\hline & (afloramientos rocosos) & $1.54 \mathrm{~km} 2$ \\
\hline $0-10$ & Nula. Erosión no perceptible en el campo & $2.34 \mathrm{~km} 2 \quad(11.5 \%)$ \\
\hline $10-20$ & Débil. Algunos regueros, procesos lentos. & $2.26 \mathrm{~km} 2 \quad(11.2 \%)$ \\
\hline $20-50$ & Moderado. Regueros frecuentes, depósitos en zonas bajas. & $6.53 \mathrm{~km} 2 \quad(32.2 \%)$ \\
\hline $50-100$ & Fuerte. Cárcavas poco desarrolladas. & $4.47 \mathrm{~km} 2 \quad(22.1 \%)$ \\
\hline$>100$ & Muy fuerte. Cárcavas y barrancos frecuentes. & $3.12 \mathrm{~km} 2 \quad(15.4 \%)$ \\
\hline
\end{tabular}

establecer las áreas prioritarias de actuación, donde se han propuesto medidas de lucha contra la erosión de suelos (Tabla 8) más restrictivas o drásticas cuanto más crítica es la zona. Consisten básicamente en cambios de uso del suelo de agrícola a forestal, restricción del pastoreo, laboreo a nivel y control de la escorrentía en zonas agrícolas.

\section{CONCLUSIONES}

- La metodología multinivel implementada en un SIG que se ha utilizado ha permitido localizar las áreas fuente de sedimentos más importantes en la cuenca del embalse de Joaquín Costa, determinar su actividad y evolución y proponer medidas correctoras que puedan rebajar la aportación de sedimentos al mismo.
- Las áreas más activas corresponden a cuencas con elevada densidad de drenaje y litológicamente con materiales poco resistentes a la erosión, elevadas pendientes y baja cubierta vegetal, localizadas en la parte media de la cuenca.

- Los procesos erosivos más graves en la cuenca son los que se producen en los badlands, de los cuales se han cuantificado pérdidas anuales del orden de $0.5 \mathrm{hm}^{3}$ en una área modelo de $1.6 \mathrm{~km}^{2}$, lo que explica que la mayor parte de la colmata-

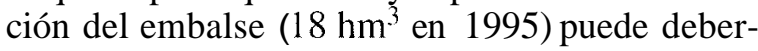
se sólo a la contribución de los badlands.

- La evolución actual de la red de cárcavas y badlands no permite preveer una estabilización natural a corto plazo, por lo cual son necesarias actuaciones adecuadas en la cuenca si se quiere disminuir la colmatación del embalse en un futuro.

- Teniendo en cuenta las limitaciones del modelo y los resultados obtenidos, no se puede

Tabla 8. Medidas de control de la erosión en laderas de la Cuenca del Congustro. Erosion control measures on the slopes of the Congustro catchment.

\begin{tabular}{|c|c|}
\hline $\begin{array}{l}\text { Unidades } \\
\text { cartográficas }\end{array}$ & Medidas propuestas \\
\hline A & $\begin{array}{l}\text { Innecesarias, en algunos casos limitación del pastoreo. sobre todo en las áreas adyacentes a badlands y } \\
\text { barrancos. }\end{array}$ \\
\hline B & $\begin{array}{l}\text { Medidas agrotécnicas (laboreo a nivel, aportaciones de materia orgánica) y control de la escorrentía con- } \\
\text { centrada en algún caso. }\end{array}$ \\
\hline $\mathrm{C}$ & $\begin{array}{l}\text { Limitación del pastoreo, laboreo de conservación, control de la escorrentía en parcelas agrícolas median- } \\
\text { te zanjas o drenajes adecuados. }\end{array}$ \\
\hline $\mathrm{D}$ & $\begin{array}{l}\text { Mejora de la cubierta mediante repoblaciones, restricción del pastoreo. control de la escorrentía mediante } \\
\text { terrazas y drenajes adecuados hacia las zonas adyacentes. }\end{array}$ \\
\hline $\mathrm{E}$ & $\begin{array}{l}\text { Hidrotecnias de estdbiiización de márgenes: diques de gabiones o mampostería seca; acotación del pasto- } \\
\text { reo, reforestación de las zonas agrícolas, control de la escorrentía. }\end{array}$ \\
\hline
\end{tabular}


aconsejar sobre la futura construcción de un nuevo embalse en la zona de Santa Liestra, el cual al estar próximo a áreas críticas detectadas por el modelo (río Rialbo y barrancos de Espluga y Bacamorta) se vería afectado negativamente.

- La propuesta de medidas de conservación tiene que realizarse en función de una cartografía de la erosión que contemple los procesos y factores erosivos en unidades cartográficas, para poder atacar sus causas.

\section{BIBLIOGRAFÍA}

DEL VAL, J. 1989. Factores de erosión. Investigación y Ciencia 1.52: 72-81.

DUNNE, T.H. y L.B. LEOPOLD. 1978. Water in environmental planning. Freeman \& Co. N.Y.

FARGAS, D. Identificació d'arees de risc d'emissió de sediments a la conca de l'embassament Joaquín Costa $(O s c a)$. PFC. ETSEA-UdL. Lleida. 78 pp.

FARGAS, D., J.A. MARTINEZ-CASASNOVAS \& R.M. POCH. 1996. Identification of critical sediment source areas at regional level. XXI General Assembly European Geophysical Society. The Hague (Holanda), 6-10 Mayo 1996. Journal of Physics and Chemistry of Earth, 22 (3-4): 355-359

GREGORY,K.J. \& V. GARDNER, 1975. Drainagedensity and climate. Z Geomorphology N.F. 19:287-298.

HORTON. R.E. 1945. Erosional development of streams and their drainage basins: Hydrophysical approach to quantitative morphology. Bull. Geol. Soc. of America, LVI: 275-370.
MARTÍNEZ-CASASNOVAS, J.A. 1994. Hydrographic information ahstraction for erosion modelling at regional level. A database perspective in a GIS environment. MsC Thesis, Wageningen Agricultural University, Wageningen.

MOPT. 1992. Propuesta del proyecto de directrices. Cuenca del Ebro. Confederación Hidrográfica del Ebro, tomo IV Resumen.

MORGAN, R.P.C. 1973. The intluence of scale in climatic geomorphology: a case study of drainage density in West Malaysia. Geografiska Annaler 55A: $107-11.5$

ONSTAD, C.A. \& C.R. FOSTER. 1975. Erosion modelling on a watershed. Transactions of the ASAE I8: 288-292.

POCH, R.M. 1993. Tècniques de conservació de sòls. Col·lecció EINES, UdL. Lleida. 82 pp.

PORTA, J., M. LÓPEZ-ACEVEDO y C. ROQUERO. 1994. Edafología para la agricultura y medio ambiente. Mundi-Prensa, Madrid.

SOIL SURVEY STAFF. 1996. Keys to Soil Taxonomy: Seventh Edition 1996. U.S. Department of Agriculture - Natural Resources Conservation Service. Washington, D.C.

STROOSNIJDER, L. y L.A.A.J. EPPINK. 1993. Principles of soil and water conservution. Lecture notes K200-500/510, Wageningen Agricultura1 University. Wageningen (Holanda). 354 pp.

WISCHMEIER, W.H. \& D.D. SMITH. 1978 Predicting rainfall erosion losses - A guide to conservation planning. USDA Agriculture Handbook num. 537. US Government Printing Office. Wa shington, DC. 58 pp. 